\title{
Original
}

\section{The Usefulness of C-arm CBCT-guided Abscess Drainage with A Flat-panel Detector}

\author{
Shinya IkedA, Noritaka SeIno, Takashi Hashizume, \\ Shouei SAI and Takehiko GoKAN
}

\begin{abstract}
Herein we report our experience with a C-arm cone beam computed tomography (CBCT)-guided abscess drainage procedure. We retrospectively examined the medical records and imaging results of patients whose abscesses were drained between 2005 and 2010 employing this system. We analyzed the technical success rate and incidence of procedure-related complications. Percutaneous abscess drainage using C-arm CBCT was performed using a flatpanel detector digital subtraction angiography (DSA) system on 104 lesions in 97 patients (55 men, 42 women) with a mean age of 64.7 (30-88) years. The drainage procedure was performed twice in 6 patients and 3 times in 2 patients, and 6 patients received 2 or more punctures at the same time. The technical success rate with this procedure was 98.1\% (102 of 104 lesions). Placement of the drainage tube was abandoned in 2 patients due to difficulty in inserting a wire into the abscess cavity in 1 case and difficulty looping the wire in the other. The incidence of procedure-related complications was $3.85 \%$ (4 of 104 lesions). Our retrospective study and review of the relevant literature revealed that the $\mathrm{C}$-arm CBCT-guided abscess drainage procedure examined was generally safe for patient use, showed a high technical success rate and low incidence of procedure-related complications, and was useful for abscesses that were inaccessible using other instruments. Although C-arm CBCT has limited contrast resolution, this disadvantage is easily overcome by comparing images with those obtained using other modalities.
\end{abstract}

Key words : radiology, IVR, drainage, C-arm CBCT, abscess

\section{Introduction}

Abscesses, which can affect various parts of the body, may be associated with infectious diseases or develop as a postoperative complication. When an abscess is refractory to antibiotics or accompanied by prolonged pyrexia or inflammation, the first-choice treatment is considered to be percutaneous drainage because this procedure is both easy to perform and minimally invasive ${ }^{1)}$. Numerous retrospective and prospective studies reported in the surgery 
and radiology literature have documented the effectiveness of percutaneous drainage, with cure rates of $62 \%-91 \%$ and with morbidity and mortality statistics equivalent to those for surgical drainage ${ }^{2-11)}$. Percutaneous abscess drainage is often guided by imaging modalities such as ultrasonography and computed tomography $(\mathrm{CT})^{12}$, and indeed, we perform drainage procedures under the guidance of C-arm cone beam (CB) CT using a flat-panel detector digital subtraction angiography (DSA) system. To assess such an approach, this study retrospectively examined the imaging results and medical records of patients whose abscesses were drained employing this system from 2005 to 2010. Herein we report on the technical success rate, incidence of procedure-related complications, and usefulness for abscesses that were inaccessible using other instruments, along with a review of the relevant literature.

\section{Materials and Methods}

The study subjects were selected from patients who had been referred to our department from April 2005 through November 2010 with a diagnosis of abscess, suspected abscess, pyogenic spondylitis, pyogenic discitis, or postoperative abscess. Percutaneous abscess drainage using a flat-panel detector DSA system by C-arm CBCT was performed for 104 lesions in 97 patients (55 men, 42 women) with a mean age of 64.7 (30-88) years. The drainage procedure was performed twice in 6 patients and 3 times in 2 patients, with 6 patients receiving 2 or more punctures at the same time. Written informed consent was obtained from all patients prior to the drainage procedure. The study protocol and scheduled article submission were approved by the ethics committee of our institution. The clinical success rate of this procedure and the incidence of procedure-related complications were examined retrospectively.The puncture procedure was performed on patients lying on the table of the DSA system. A radiopaque scale was fixed to the patient's skin. Guided by cross-sectional images and three-dimensional (3D) images obtained with the C-arm CBCT, the skin entry and target points were marked on the computer workstation. The patient's skin was then also marked according to the calibration scale. Actual entry points were determined by referring to the calibration markings on the skin and in the C-arm display ${ }^{13)}$, and mapping the puncture route from the skin entry to target point on the computer workstation. The puncture route distance was measured by placing the flat-panel detector in the 90-degree position relative to the puncture route. Then, the angular position was adjusted to assure that a bull's eye view was achieved on the computer screen by aligning the target and skin entry points obtained by $3 \mathrm{D}$ imaging. The detector panel was adjusted to that angle, and the patient's table was moved so that the actual skin entry point and the puncture route had the same direction as the rotating axis of the flat-panel detector. In this setting, puncture was performed with a 19-gauge ELASTER needle (HAKKO Co., Ltd.) after using the imaging guidance to align the anterior and posterior ends of the needle. Then, a 0.038inch Bentson wire (MEDICOS Hirata Co., Ltd.) was looped within the abscess cavity, and a UreSil (Sheen Man Co., Ltd.) catheter was placed with the help of a guidewire ${ }^{14)}$. 
Table 1.

\begin{tabular}{lr}
\hline Clinical success rate & $102 / 104 \operatorname{cases}(98.1 \%)$ \\
Incidence of procedure-related & $4 / 104 \operatorname{cases}(3.85 \%)$ \\
\hline
\end{tabular}

Table 2 .

\begin{tabular}{lc}
\hline Primary disease & lesions \\
\hline Iliopsoas muscle abscess & 34 \\
Spondylodiscitis & 18 \\
Hepatic abscess & 10 \\
Osteomyelitis & 7 \\
Empyema & 6 \\
Digestive tract perforation & 5 \\
Postoperative abscess & 13 \\
Infectivity bulla & 1 \\
Perianal abscess & 1 \\
Tumor & 4 \\
Peritonitis & 1 \\
Infection of renal hematoma & 1 \\
Otherwise & 3 \\
\hline
\end{tabular}

Table 3 .

\begin{tabular}{ll}
\hline Past history of operation & \\
\hline Ovarian cancer & 3 \\
Bile duct cancer & 2 \\
Transverse colon cancer & 1 \\
Ascending colon cancer & 1 \\
Pancreatic cancer & 1 \\
Ureter cancer & 1 \\
Prostate cancer & 1 \\
Bladder cancer & 1 \\
Appendicitis & 1 \\
Infectious aortic aneurysm & 1 \\
\hline
\end{tabular}

\section{Results}

The technical success rate with this procedure was 98.1\% (102 of 104 lesions). Placement of the drainage tube was abandoned in two patients because of difficulty inserting the wire into the abscess cavity in one case and difficulty looping the wire in the other. The incidence of procedure-related complications was 3.85\% (4 of 104 lesions; Table 1). One patient developed bloody sputum when the catheter was placed in an empyema. In another patient, the guidewire remained in the abscess cavity, and the remaining two patients became hypotensive, probably due to vagal reflex resulting from hematoma and pain, respectively, at the sites of local anesthesia. The diagnoses (104 lesions in total) included iliopsoas muscle abscess (34 lesions), spondylodiscitis ${ }^{18)}$, hepatic abscess ${ }^{10)}$, osteomyelitis ${ }^{7)}$, empyema ${ }^{6}$, digestive tract perforation ${ }^{5)}$, and postoperative abscess ${ }^{13)}$ (Table 2). Primary diagnoses of patients with postoperative abscesses were transverse colon cancer (1 case), ascending colon cancer $^{1)}$, pancreatic cancer $^{1)}$, ureter cancer $^{1)}$, prostate cancer $^{1)}$, bladder cancer ${ }^{1)}$, appendicitis $^{1)}$, infectious-aortic aneurysm ${ }^{1)}$, bile duct cancer ${ }^{2)}$, and ovarian cancer $^{3)}$ (Table 3). Abscess cavities were clear in 88 lesions, whereas the other 16 showed irregular soft tissue shadows. Mean preoperative lesion size was $56.4 \mathrm{~mm}$ (Table 4). We used an $8 \mathrm{Fr}$ catheter in approximately half of our cases $(58 / 117,49.6 \%)$, followed by $10 \mathrm{Fr}(23.1 \%), 6 \mathrm{Fr}(12.0 \%)$, and $12 \mathrm{~F}$ (5.1\%) catheters (Table 5). 
Table 4.

\begin{tabular}{lcc}
\hline Clinical background & \\
\hline Size of abscess (maximum length) & $7 \sim 144 \mathrm{~mm}($ mean $56.38 \mathrm{~mm})$ \\
\hline Appearance of abscess & Monocystic lesion & 47 \\
& Polycystic lesion & 41 \\
& Irregular soft tissue shadows & 16 \\
\hline
\end{tabular}

Table 5 .

\begin{tabular}{lc}
\hline Catheter size & \\
\hline $6 \mathrm{Fr}$ & $14(12.0)$ \\
$7 \mathrm{Fr}$ & $0(0)$ \\
$8 \mathrm{Fr}$ & $58(49.6)$ \\
$10 \mathrm{Fr}$ & $27(23.1)$ \\
$12 \mathrm{Fr}$ & $6(5.1)$ \\
Otherwise & $3(2.6)$ \\
Unknown & $9(7.6)$ \\
\hline & $117(100 \%)$ \\
\hline
\end{tabular}

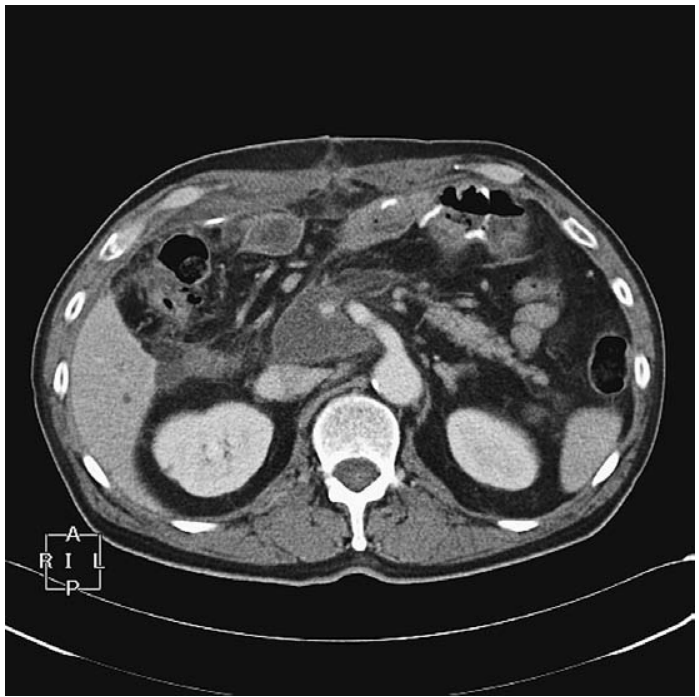

Fig. 1. Computed tomography showing an abcess that developed after pancreatoduodenectomy. The abscess has an enhanced wall and is located around the superior mesenteric artery.

\section{Case Presentation}

A 70-year-old man with an intraductal papillary mucinous tumor underwent pancreatoduodenectomy. Fluid subsequently collected in the area surrounding the superior mesenteric artery (Fig. 1), and the patient was referred to our department for drainage of a suspected postoperative abscess. The patient was placed in a semi-lateral position on the table of the imaging system (Fig. 2). Before starting the drainage procedure, a radiopaque scale was fixed to the skin directly over the center of the fluid collection, aided by the CT scan. Then, C-arm CBCT was initiated (Fig. 3). Since the lesion was located in a central part of the trunk, it was difficult to plan a puncture route that to avoid vascular structures and the intestinal tract based on the cross-sectional and 3D images. Therefore, we planned a relatively safe transhepatic route using 3D images on the computer workstation, and performed the puncture procedure while monitoring the location of the needle with the C-arm CBCT. 


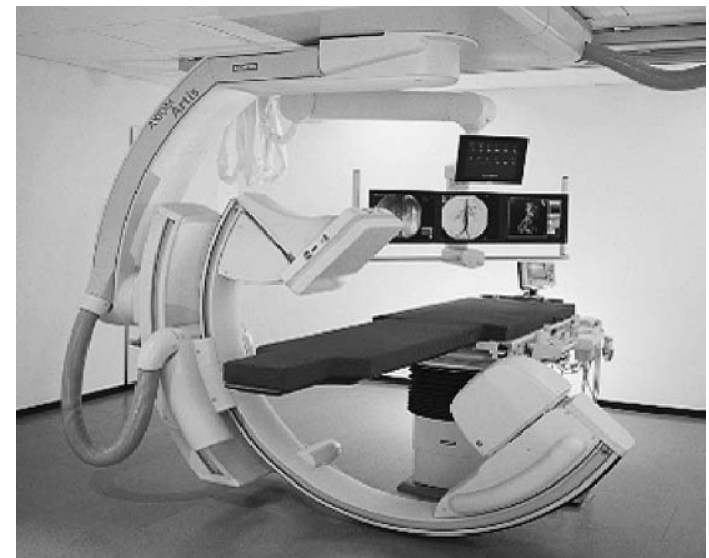

Fig. 2. The DSA system used features a flat-panel detector and enables flexible positioning around the patient on the table.

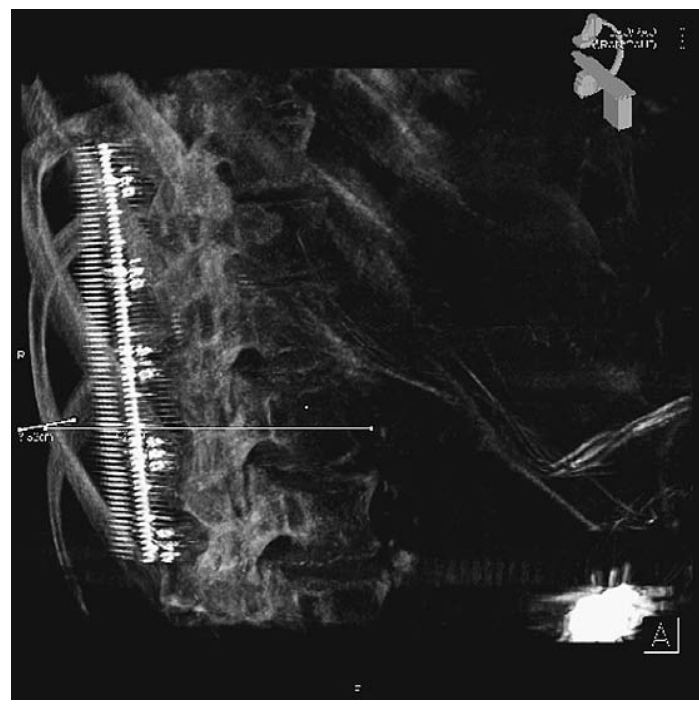

Fig. 3. C-arm CBCT detected soft tissues around the superior mesenteric artery that seemed to be the abscess we observed in the preoperative CT.

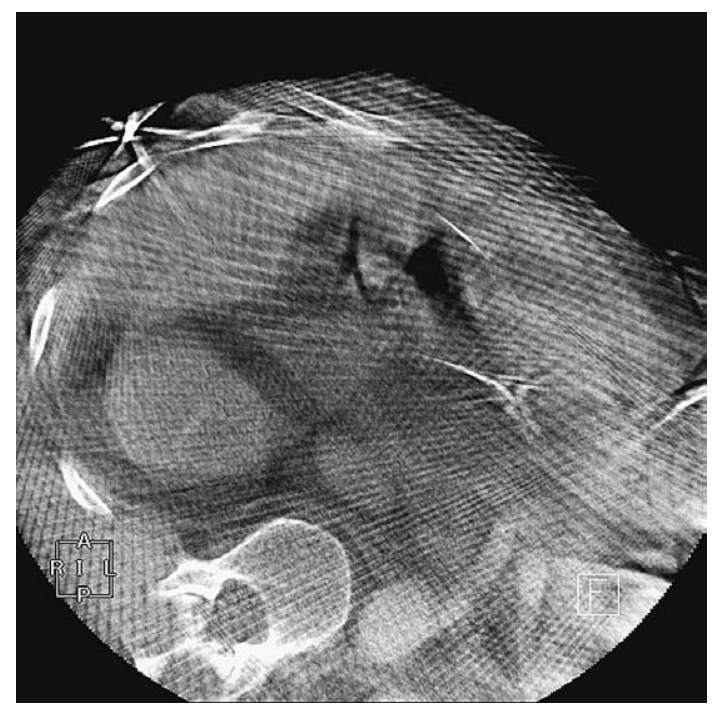

Fig. 4. The puncture route from the skin entry to target point was mapped on the computer workstation. The puncture route distance was measured by placing the flat-panel detector in the 90-degree position relative to the puncture route.

As mentioned above, a puncture route was determined on the computer workstation and the puncture route distance was measured (Fig. 4). Then, the patient's bed was moved so that the actual skin entry point and the puncture route had the same direction as the flatpanel rotation. A local anesthetic (1\% lidocaine) was injected at the skin entry point. The direction of the 19-gauge ELASTER needle $(20 \mathrm{~cm})$ was adjusted to assure that the ante- 


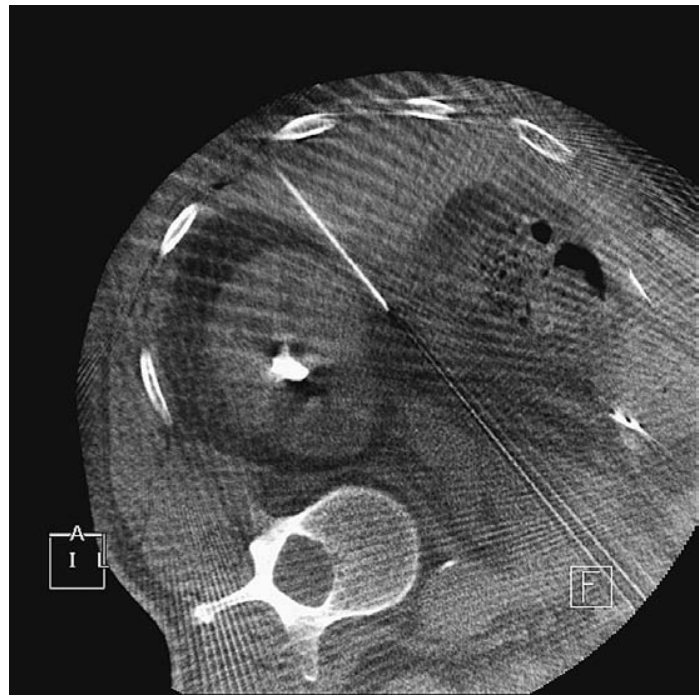

Fig. 5. If we want to confirm the location of needle, it is easy to verify by using C-arm CBCT.

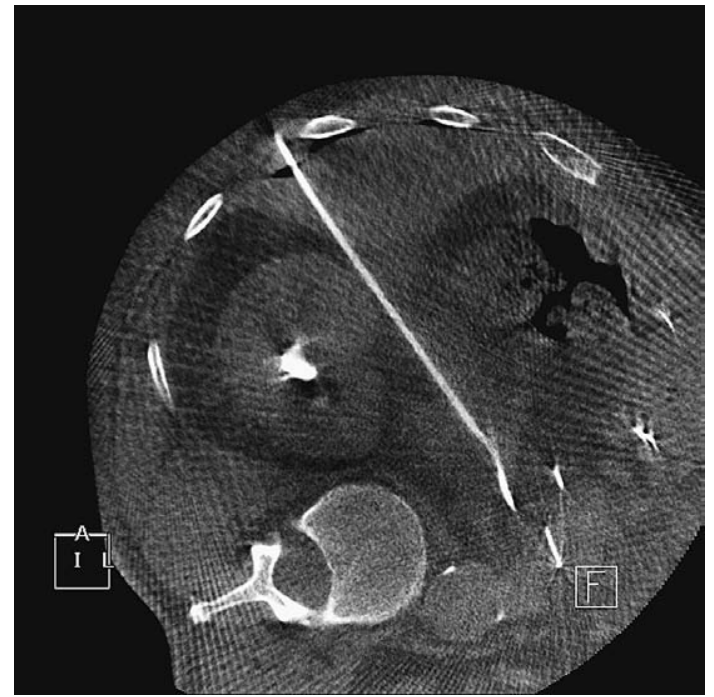

Fig. 6. A guidewire (0.038-inch Bentson type) was inserted into the abscess cavity and formed into a loop. Then, a drainage catheter $(6 \mathrm{Fr}$ Uracil catheter) was placed in the abscess around the superior mesenteric artery.

rior and posterior ends of the needle were superimposed on the fluoroscopic images, while avoiding sites just below the right 10th rib. For the transhepatic puncture, the puncture needle was inserted gradually, and the location of the needle was confirmed by the C-arm CBCT (Fig. 5). When the puncture needle was advanced to the measured target point on the computer workstation, C-arm CBCT was performed to confirm that the anterior end of the needle tip had reached the fluid. The inner needle was removed, and a small amount of fluid was withdrawn by suction. Then, a guidewire (0.038-inch Bentson type) was inserted into the abscess cavity and formed into a loop. The puncture route was expanded with a dilator, and a drainage catheter (8Fr Uracil catheter) was placed in the abscess with guidewire assistance (Fig. 6). A small amount of fluid was withdrawn by suction, and the procedure was completed.

\section{Discussion}

Percutaneous drainage under imaging guidance is often the first-choice treatment for abscesses because it is relatively easy to perform and minimally invasive ${ }^{1,12)}$. Each of the imaging methods used has advantages and disadvantages. Ultrasonography has the advantages of high tissue resolution, real-time observation, and no radiation exposure. On the other hand, it has the disadvantages of variable image quality according to the skill of the operator, difficulty visualizing lesions surrounded by bone or air and deep lesions in the trunk, and limitations in spatial and contrast resolution for obese patients ${ }^{15)}$. CT fluoroscopy 
has the advantage of high spatial resolution which allows relatively good visualization of small lesions, but may have a limitation in that puncture is sometimes difficult in the craniocaudal direction due to the restricted tilt angle of the detector ${ }^{15}$. In this context, C-arm CBCT has the following advantages : 1) it is possible to set the puncture line easily on 3D images by processing the volume data obtained with the C-arm CBCT on the 3D workstation, and real-time observation of the puncture line is possible by performing the puncture under fluoroscopic guidance while acquiring a Bull's eye view by aligning the skin entry point and the target point. This approach facilitates avoiding accidental puncture;2) it is possible to confirm the needle tip location and surrounding organs by performing $\mathrm{C}$-arm CBCT as needed. This step assures safety for both the patient and the operator; and, 3) the same procedure can be carried out with only the C-arm CBCT and 3D workstation and does not require a special test room for $\mathrm{CT}^{16)}$. The disadvantages of $\mathrm{C}$-arm $\mathrm{CBCT}$ are the possibly limited visualization of low-density lesions such as abscesses because the contrast resolution of $\mathrm{C}$-arm $\mathrm{CBCT}$ is generally restricted to approximately 10 to $20 \mathrm{Hu}^{17)}$, and that the puncture plan on the workstation may not coincide exactly with the actual puncture due to body movements after obtaining the 3D volume data. Further improvements in contrast resolution are needed to address the former problem ${ }^{18)}$, but in the present study, we were able to maximize puncture route safety by comparing the images on the workstation with those of preoperative CT or by performing contrast-enhanced CT for patients showing obscure boundaries with vessels and surrounding organs. To address the latter problem, we attempted to place the patient in a sustainable posture preoperatively, and to approximate the actual puncture to the estimated puncture plan by synchronizing with breathing movements in patients undergoing chest to upper abdominal puncture ${ }^{19)}$. The technical success rate with this procedure in the present study was approximately 98\%. The wire manipulation difficulties encountered in these two cases were due to a very small abscess cavity measuring approximately $12 \mathrm{~mm}$ in diameter on preoperative CT and increased intensity of the cavity wall, respectively. However, the puncture process itself was completed even in these cases under C-arm CBCT guidance. The incidence of procedure-related complications in the present study was 4\% (4 of 104 lesions), lower than in other reports ${ }^{16-17,20-22)}$. The two affected cases had subcutaneous hematoma and vagal reflex, respectively. One patient developed bloody sputum due to percutaneous pulmonary puncture, considered to be an unpredictable complication. There were no accidental punctures of other organs.

\section{Conclusion}

We reported the technical success rate and incidence of procedure-related complications for C-arm CBCT-guided abscess drainage, and assessed the usefulness of such a procedure for abscesses that were inaccessible using other instruments. We also reviewed the relevant literature. Although $\mathrm{C}$-arm $\mathrm{CBCT}$ has limited contrast resolution, this disadvantage is easily overcome by comparing images with those obtained using other modalities. We have found 
this procedure to generally be safe.

\section{References}

1) Golfieri R and Cappelli A : Computed tomography-guided percutaneous abscess drainage in coloproctology: review of the literature. Tech Coloproctol 11:197-208 (2007)

2) Lang EK, Springer RM, Glorioso LW 3rd and Cammarata CA : Abdominal abscess drainage under radiologic guidance : causes of failure. Radiology $159:$ 329-336 (1986)

3) Gazelle GS and Mueller PR : Abdominal abscess. Imaging and intervention. Radiol Clin North Am 32 : 913-932 (1994)

4) Bouali K, Magotteaux P, Jadot A, Saive C, Lombard R, Weerts J, Dallemagne B, Jehaes C, Delforge M and Fontaine F: Percutaneous catheter drainage of abdominal abscess after abdominal surgery. Results in 121 cases. J Belge Radiol $76: 11-14$ (1993)

5) vanSonnenberg E, Casola G, Ho M, Neff C, Varney RR, Wittich GR, Christensen R and Friedman PJ : Difficult thoracic lesions: CT-guided biopsy experience in 150 cases. Radiology 167:457-461 (1988)

6) Lambiase RE, Deyoe L, Cronan JJ and Dorfman GS: Percutaneous drainage of 335 consecutive abscesses: results of primary drainage with 1-year follow-up. Radiology 184:167-179 (1992)

7) Rothlin MA, Schob O, Klotz H, Candinas D and Largiader F : Percutaneous drainage of abdominal abscesses: are large-bore catheters necessary? Eur J Surg 164: 419-424 (1998)

8) vanSonnenberg E, Ferrucci JT Jr, Mueller PR, Wittenberg J and Simeone JF : Percutaneous drainage of abscesses and fluid collections: technique, results, and applications. Radiology 142 : 1-10 (1982)

9) Bufalari A, Giustozzi G and Moggi L: Postoperative intraabdominal abscesses: percutaneous versus surgical treatment. Acta Chir Belg $96: 197-200$ (1996)

10) vanSonnenberg E, Wing VW, Casola G, Coons HG, Nakamoto SK, Mueller PR, Ferrucci JT Jr, Halasz NA and Simeone JF : Temporizing effect of percutaneous drainage of complicated abscesses in critically ill patients. AJR Am J Roentgenol 142: 821-826 (1984)

11) Jaffe TA, Nelson RC, Delong DM and Paulson EK : Practice patterns in percutaneous image-guided intraabdominal abscess drainage : survey of academic and private practice centers. Radiology 233 : 750-756 (2004)

12) Kurosaki I, Takagi K, Hatakeyama S, Nagai K, Sekiya M, Seki K, Koyama T, Tsukada K and Hatakeyama K: Right hepatectomy for pyogenic liver abscesses with true multiloculation. J Gastroenterol 32 : 105-109 (1997)

13) Hashimoto T, Seino N, Honda M, Hashizume T, Shinjo H, Ogiya $Y$ and Gokan $T$ : Flat detector C-armCT for interventional radiology : new development with DynaCT. J Tomogr 33 : 126-133 (2006) (in Japanese)

14) Leschka SC, Babic D, El Shikh S, Wossmann C, Schumacher M and Taschner CA: C-arm cone beam computed tomography needle path overlay for image-guided procedures of the spine and pelvis. Neuroradiology $\mathbf{5 4}: 215-223(2012)$

15) Higashihara $\mathrm{H}$, Osuga $\mathrm{K}$, Maeda $\mathrm{N}$, Tomoda $\mathrm{K}$, Mikami $\mathrm{K}$, Chun HJ, Nakazawa $\mathrm{T}$ and Nakamura $\mathrm{H}$ : Initial experience of a novel cone-beam CT guided percutaneous drainage for abscesses in the trunk. Jpn J Intervent Radiol 24 : 146-149 (2009) (in Japanese)

16) Braak SJ, van Strijen MJ, van Leersum M, van Es HW and van Heesewijk JP : Real-Time 3D fluoroscopy guidance during needle interventions: technique, accuracy, and feasibility. AJR Am J Roentgenol 194 : W445-W451 (2010)

17) Hashimoto $T$, Honda $M$, Seino $N$, Hashizume $T$, Hayano $D$, Kubota $M$, Hirose $M$, Nobusawa $H$ and Gokan T: Clinical usefulness of C-armCT for interventional radiology. Jpn Dtsch Med Ber 51:271-282 (2006) (in Japanese)

18) Akpek S, Brunner T, Benndorf $G$ and Strother $C$ : Three-dimensional imaging and cone beam volume $C T$ in C-arm angiography with flat panel detector. Diagn Interv Radiol 11 : 10-13 (2005)

19) Morimoto M, Numata K, Kondo M, Nozaki A, Hamaguchi S, Takebayashi S and Tanaka K : C-arm cone beam CT for hepatic tumor ablation under real-time 3D imaging. AJR Am J Roentgenol 194: W452-W454 (2010) 
20) Lagana D, Carrafiello G, Mangini M, Ianniello A, Giorgianni A, Nicotera P, Fontana F, Dionigi G and Fugazzola C: Image-guided percutaneous treatment of abdominal-pelvic abscesses: a 5-year experience. Radiol Med 113 : 999-1007 (2008)

21) Thomas J, Turner SR, Nelson RC and Paulson EK: Postprocedure sepsis in imaging-guided percutaneous hepatic abscess drainage: how often does it occur? AJR Am J Roentgenol 186 : 1419-1422 (2006)

22) Bakal CW, Sacks D, Burke DR, Cardella JF, Chopra PS, Dawson SL, Drooz AT, Freeman N, Meranze SG, Van Moore A Jr, Palestrant AM, Roberts AC, Spies JB, Stein EJ, Towbin R and Society of Interventional Radiology Standards of Practice Committee: Quality improvement guidelines for adult percutaneous abscess and fluid drainage. J Vasc Interv Radiol 14: S223-S225 (2003)

[Received December 26, 2011 : Accepted January 13, 2012] 